\title{
Laser-Induced Breakdown Spectroscopy as Diagnostics for Plasma-Wall Interactions Monitoring in Tokamaks
}

\author{
P. GĄSIOR \\ IFPILM, Hery 23, 01-497 Warszawa, Poland \\ Doi: $10.12693 /$ APhysPolA.138.601 \\ *e-mail: pawel.gasior@ifpilm.pl
}

\begin{abstract}
Plasma-wall interactions in tokamaks inflict local erosion which often leads to the deposition of the removed material in distant areas, mainly lower temperature zones of the divertor. This phenomenon is accompanied with the accumulation of fuel particles in deposits, which is called fuel retention, and in future will be particularly important in the case of radioactive tritium. The fuel inventory needs to be monitored which therefore requires a measurement method which, due to the harsh thermonuclear conditions, must be contactless and highly resistant to electromagnetic interference. The method which satisfies these prerequisites is laser-induced breakdown spectroscopy basing on the remote acquisition of the optical signal which may be induced by a laser head installed on a remotely controlled conveyor or even outside the device. The presented method was originally applied for the carbon-based plasmafacing components which manifested intensive chemical erosion and therefore unacceptably high rate of fuel retention. Nevertheless, the method proved its capability for these conditions and furthermore was found to be even more advantageous for plasma-facing components manufactured with the use of mixed materials (beryllium and tungsten) since it allowed for the monitoring of the material transport to the divertor. At the IPPLM, research on laser-induced breakdown spectroscopy originated from the former investigation of the possibility of employing pulse lasers in the laser cleaning of the fuel inventory. The recent work outlines the progress of the method: starting from a spin-off application for monitoring of the laser cleaning process, through the adaptation to mixed material components, to the advanced alternatives such as dual pulse or calibration-free laser-induced breakdown spectroscopy which are currently under development in EUROfusion collaboration with such international partners as ENEA Frascati, FZJ and others.
\end{abstract}

topics: thermonuclear fusion, plasm-wall interactions, laser-induced breakdown spectroscopy, LIBS

\section{Introduction}

The domestication of thermonuclear fusion has a convincing potential for fulfilling the energy needs of humankind. However, it requires enormous interdisciplinary scientific effort to be attained. Among competitive approaches, the magnetic confinement fusion $(\mathrm{MCF})[1,2]$ is considered to be the most promising. Being treated as the mainstream of the fusion research, it consumes most of the scientific effort as well as the majority of the finances in the paramount ITER project [3].

Among various challenges faced by the MCF, gaining control over plasma-wall interactions (PWI) belongs to the crucial ones [4-6]. These interactions have an impact not only on the safety and wall-maintaining issues in both tokamaks $[1,2]$ and stellarators $[7,8]$ but they may also influence plasma performance by leading to disastrous radiation losses or even inducing disruptions in tokamaks resulting in a damage to the device. Taking into account the above-mentioned issues, it becomes obvious how relevant are methods for observation, monitoring, quantification and control of the phenomena occurring on the plasma and the wall boundary. Among the quantities which are of foremost importance, the chemical composition of the wall may be distinguished since it constitutes an evidence of processes responsible for erosion or deposition. These processes, in fact, have essential impact on plasma-facing components' performance.

The development of methods may seem to be a technical rather than a scientific issue, however, the diagnostic systems for PWI must comply with very strict requirements which are unlike any requirements for measurement and control systems present in industrial or even scientific installations. Firstly, they must withstand a unique combination of electromagnetic fields, radiation and high temperature which have a deteriorating impact on most types of the measurement systems. Next, they cannot interfere with sophisticated systems of plasma control and heating. Further, the system should employ a no-contact and no-damage method for the wall chemical composition analysis. A method which fulfils all these requirements is laser-induced breakdown spectroscopy (LIBS) [9]. 


\section{Basic LIBS features in the context of PWI}

LIBS is an analytical method which applies a high-power laser pulse to induce a miniature amount of plasma. Then, its emission spectra are observed that are composed of a fingerprint lines which are originating from the chemical composition, since each chemical element has a unique set of the lines in the wavelength domain. The laser beam is focused to a power density in the range from several to tens $\mathrm{GW} / \mathrm{cm}^{2}$ and the laser-produced plasma has a temperature exceeding $1 \mathrm{eV}$ and electron density in the range of $10^{17} \mathrm{~m}^{-3}$. As the laser radiation sources, the nanosecond pulsed lasers are most popular due to their low cost and straightforward and flexible operation; however, with the development of the femtosecond lasers, they also gain popularity because of their possibility of obtaining "cleaner ablation". The scientific LIBS spectrometers can be essentially divided into two basic subgroups: narrow-band with high spectral resolution and broad-band but with lower resolution. Most often, both types use ICCD detectors to obtain satisfactory signal levels even in the case of observation of small amounts of plasma from distant locations.

Laser-induced breakdown spectroscopy hitherto has been successfully applied in a broad range of scientific investigations: in soil analysis [10-12], metallurgy [13, 14], coal combustion [15, 16], biotechnology and medicine [17], cultural heritage preservation [18], investigation of Martian rocks in Curiosity's [19] mission and many more. Its application in thermonuclear fusion has been taken into consideration thanks to its capability of the contactless operations (in some applications laser-induced plasma has been under observation from a few hundred meters) and no need for installation of troublesome additional systems in the tokamak chamber (which would pose a risk of interference with other systems). Since it employs optical signals (both for inducing the plasma and for its observation), it is expected not to be distorted by harsh conditions caused by the impact of strong magnetic fields, radiation or high and low temperatures. Moreover, since it is one of laser techniques which use equipment of very high quality, it offers great reliability and efficiency and may be combined with akin application, as for example co-deposit and fuel removal. In fact, the research on LIBS at the IPPLM originated from the investigation of fuel removal from in-vessel tokamak components.

\section{LIBS development at the IPPLM}

\subsection{Co-deposit removal}

The experiment on the fuel removal from in-vessel tokamak components had started at the IPPLM in 2005, soon after joining EURATOM and accessing projects on fusion development coordinated by the European Fusion Development Agreement (EFDA). The first task was aimed at the investigation of the fuel and co-deposit removal from a sample of a limiter (ALT-II) of the TEXTOR tokamak by means of nanosecond laser pulses provided by NL303HT laser operating at $1064 \mathrm{~nm}$ and delivering $3.5 \mathrm{~ns}$ pulses of up to $0.8 \mathrm{~J}$ energy with the repetition rate of up to $10 \mathrm{~Hz}$. The sample was made of a Toyo Tanso, IG-430U and - due to its operation inside the tokamak - it was covered with the $40-60 \mu \mathrm{m}$ thick co-deposit consisting of carbon and deuterium. The target was installed in a vacuum under the pressure of $5 \times 10^{-5}$ mbar. As the diagnostics of the interaction of the pulses with the target, ion collectors (ICs) and electrostatic ion energy analyser (IEA) were applied. The ICs installed at $71 \mathrm{~cm}$ from the target provided the velocity distribution of the incident plasma ions by the time-of-flight method. The IEA, which is a more sophisticated equipment, served for characterisation of the chemical composition of the target during the subsequent laser pulses, thus it was expected to be capable of monitoring the fuel removal process. It was assumed that with the increase of the number of pulses delivered into a single spot, the IEA should record the decreasing amount of deuterium, which was the significant constituent of the co-deposit layer. During the experiment, various scenarios of the co-deposit removal were tested and compared with the removal of an original graphite sample (Ringsdorff EK98).

The results of the experiment [20] confirmed the possibility of removal of the co-deposit as well as allowed to determine the laser-pulse parameters which could ensure effective layer removal with the minimised risk of the substrate damage. On the other hand, the research discovered some crucial drawbacks of the investigated approach: a very low speed of the process resulting from a low average power of the applied Nd:YAG laser and the inadequateness of application of the IEA in tokamak due to its bulky construction and low temporal resolution (it could operate with the resolution rate lower than $0.1 \mathrm{~Hz}$ ). Further attempts of dealing with these problems enlightened two further directions of research: optimisation of the co-deposit removal with the use of a high-power, high repetition fibre laser and development of the diagnostics for fuel removal with the LIBS method. The research on the latter, which is the main topic of the paper, surpassed its original application and developed as a universal technique for the first wall monitoring in tokamak devices.

\subsection{Rudimentary LIBS experiments}

After realizing that LIBS can be considered useful, two preliminary experiments have been performed to corroborate this presumption. The first of them was carried out at the IPPLM in collaboration with Prof. W. Szymanski's team (Institute of Fundamental Technological Research) 


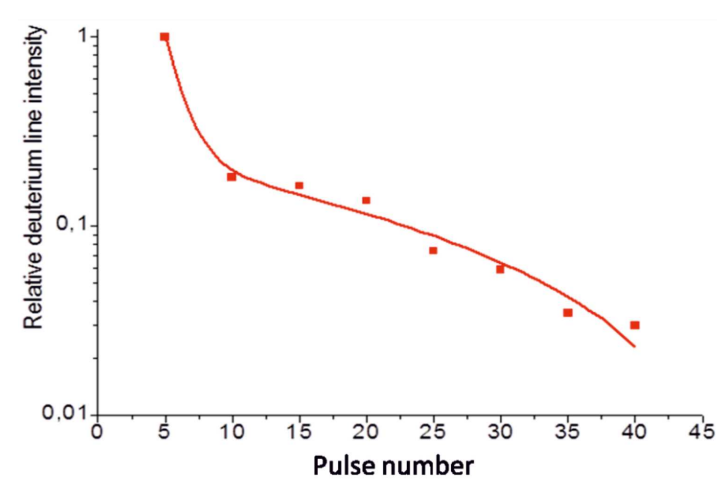

Fig. 1. Relative deuterium intensity vs. the number of laser pulses.

and, essentially, was a repetition of the original experiment with the TEXTOR limiter tile but with the ion time-of-flight diagnostics replaced with a narrowband spectrometer for the observation of the carbon and deuterium lines located in 650-670 nm wavelength range. The spectrometer was equipped with a grating with the reciprocal dispersion of $\approx 13 \mathrm{~A} / \mathrm{mm}$. The width of the entrance slit was $150 \mu \mathrm{m}$ and the plasma plume, $3 \mathrm{~mm}$ in front of the target, was imaged on the entrance slit using a $180 \mathrm{~cm}$ focal length camera lens. Based on the Stark broadening of the C(III) $(569.6 \mathrm{~nm})$, the electron density was estimated to be $8.5 \times 10^{23} \mathrm{~cm}^{-3}$ which was enough to satisfy the LTE condition. The electron temperature was calculated as $3.8 \times 10^{4} \mathrm{~K}$ with the use of the ratio of C(III) (569.6 nm) and C(II) 566.2 ionic lines.

Apart from characterizing basic plasma parameters as the electron temperature and concentration, the research attained its essential goal: it proved LIBS to be a correct method for monitoring the process of the fuel/co-deposit removal [21]. However, the signal needed to be integrated for each of the 5 subsequent laser pulses; at that time it was not a problem, since the removal process lasted for about 40 pulses. The results allowed for the assessment of the relative deuterium contents based on the ratio between the intensities of deuterium and carbon lines which is shown in Fig. 1.

The second experiment was performed in collaboration with Dr. E. Sadowska-Składnik (The Andrzej Sołtan Institute for Nuclear Studies) and became an attempt at the observation of the tungsten spectral lines with the use of the broadband spectrometer Mechelle 900. It was an important test since it was a time when tungsten had been gaining popularity as a material in thermonuclear technology, namely, as a substitute for CFC in the International Thermonuclear Experimental Reactor (ITER) divertor which was aimed at eliminating chemical erosion. Observation of the tungsten lines was a big challenge for two reasons: Mechelle 900 was not equipped with an image intensifier, thus it needed integration over many spectra and the element itself has got a very complicated spectrum consisting of numerous lines. Nevertheless, the experiment performed in conditions similar to the experiment on the TEXTOR sample resulted in obtaining correct tungsten spectra. Moreover, such plasma parameters as $T_{e}$ and $n_{e}$ have been estimated (based on the simulated Saha-LTE spectrum from the NIST database) and compared with those based on timeof-flight diagnostics (which have been applied simultaneously) which yielded consistent results [22]. Admittedly, due to fluctuations of the signal (used for the estimation of the error calculation), the results had relatively high uncertainties, i.e., $1 \mathrm{eV}$ to $1.2 \mathrm{eV}$ for the temperature, and $(0.6-1) \times 10^{17} \mathrm{~cm}^{-3}$ for $n_{e}$.

\subsection{Investigation of TEXTOR and AUG samples with the use of Mechelle 5000}

After the successful preliminary experiments, it was recognized that the application of a broadband spectrometer is a way-to-go and a decision was taken to purchase Mechelle 5000 equipped with ISTAR ICCD camera which made it possible to make measurements with sophisticated timing and excellent temporal resolution. As the first step, some experiments on the TEXTOR limiter sample have been performed for comparison with those which were performed with the use of the narrowband spectrometers. The measurements brought excellent results providing high quality spectra, illustratively showing the removal of the co-deposit from the sample after approximately 40 laser pulses. The results for the signal accumulated during the first 5 pulses (top) and the last 5 pulses (bottom) are presented in Fig. 2. On the top one,
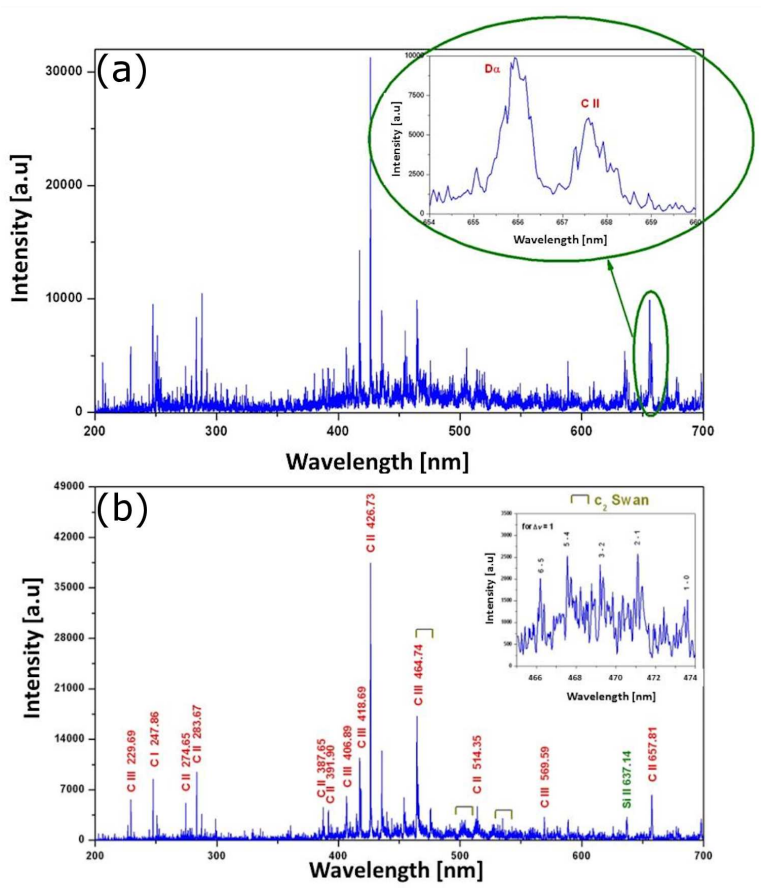

Fig. 2. Initial LIBS spectrum (top) and the spectrum after delivering 40 laser pulses onto the TEXTOR co-deposited limiter sample (bottom). 
the deuterium line contribution is clearly visible, whereas on the bottom one the line is completely diminished and simultaneously the spectrum consists of only carbon lines together with a molecular Swan band which are distinctive for carbon.

As it was already mentioned, tungsten has gained significance about the same time, hence the next experiments were performed on the tungstencovered components of AUG divertor which, due to the plasma operation in the device, were loaded with deuterium. The tungsten layer had an expected thickness of a few micrometers and the deuterium was expected to be accumulated even at the sub-micrometer depth. This allowed to decrease the number of the accumulated spectra and obtain a correct spectrum from a single spot. After the optimization of the observation time and delay as well as the focusing of the laser beam and observation geometry, a satisfactory signal level was achieved after 2 signal accumulations (i.e., signal to noise ratio better than $20 \mathrm{~dB}$ ). It turned out to be efficient for observation: first, of the deuterium removal during 2 laser pulses and further of the whole tungsten layer after 6 pulses [23]. The results were confirmed by the simultaneously applied IEA which was important from the perspective of application of LIBS in the so-called mixed materials.

\subsection{LIBS for mixed materials in PWI and chemical profiling}

The replacement of CFC in ITER divertor has strongly reduced the concern on the chemical erosion and co-deposition with carbon. However, it was promptly understood that the new design may still suffer from co-deposition with beryllium. Admittedly less intensive, this type of interaction may still become significant due to a large surface of the Be wall foreseen for the next-step fusion reactors. Therefore, it was recognized that some diagnostics must be developed for monitoring material migration from the main chamber to the divertor. Since LIBS was already a promising method, research began on adopting it to the mixed-material conditions. The major obstacle was the lack of possibility of carrying out any research with beryllium at the IPPLM. Nevertheless, it was soon proved that in the LIBS investigation it may be replaced with aluminum.

The research on mixed-materials LIBS at the IPPLM started with the investigation of the samples of mixed hydrogenated layers containing different mixes of $\mathrm{Al}, \mathrm{W}$ and $\mathrm{C}$ prepared on the aluminum substrates by means of epitaxy at the IETM, Warsaw. The results showed straight correlation between the chemical concentration of the elements and the intensity of the lines in spectra [23]. To characterize the ratios, a synthetic contents coefficient has been proposed which was expressed by:

$$
\mathrm{Scc}_{e}=\frac{\sum_{i} I_{\mathrm{ie}}}{\sum_{a} I_{\mathrm{ae}}},
$$
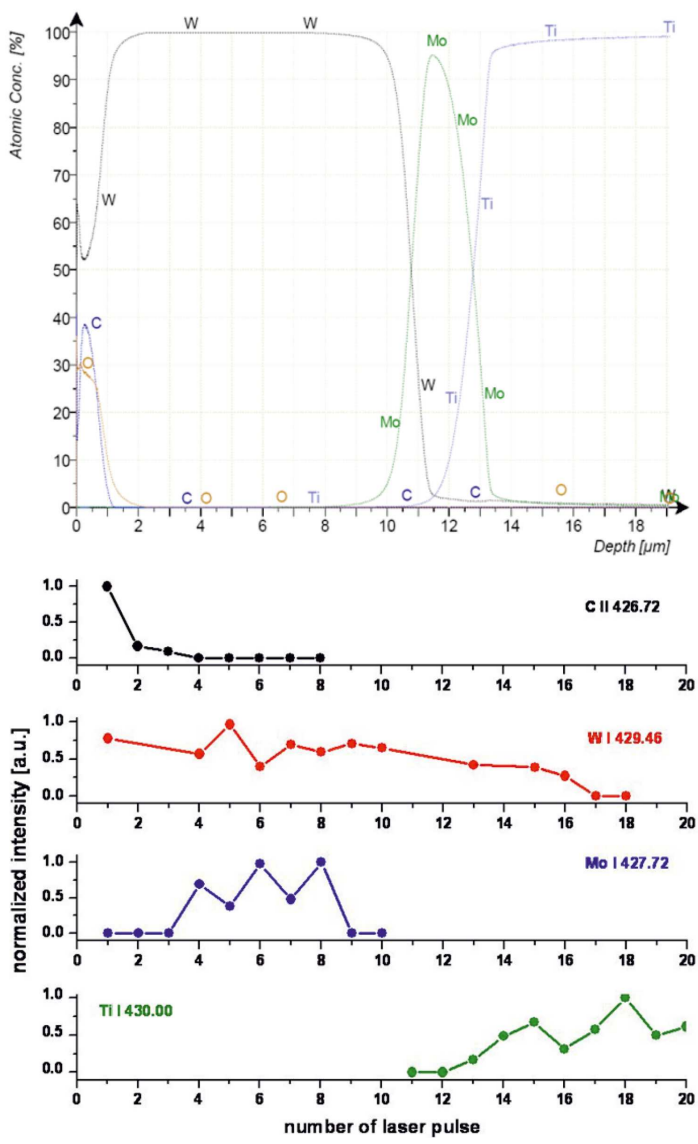

Fig. 3. GDOS chemical analysis of a calibrated sample (top) and the results of LIBS chemical profiling (bottom).

where $\mathrm{Scc}_{e}$ is a synthetic contents coefficient of an element $e, I_{\text {ie }}$ are the intensities of the lines of the element under investigation, and $I_{a e}$ are the intensities of all elements in the spectrum. To be considered, the line intensity should have exceeded $10 \%$ of the intensity of the strongest line of a given element. Another important finding of this research was that the hydrogen retention as well as its removal rate had not depended strongly on the ratios of the elements in the mixed layers.

The findings of the research on mixed material layers opened the possibilities of performing chemical profiling of the samples, i.e., monitoring the chemical composition in dependence on the depth which was correlated with the number of pulses. This kind of research was performed for numerous calibrated samples which had been provided in the framework of many EFDA and further EUROfusion tasks. Sample results of such investigations are presented in Fig. 3 which shows chemical profiling of the elements in a calibrated sample prepared by magnetron sputtering.

On the left, there is a GDOS profile of the sample and on the right, the normalized intensity of the lines attributed to its chemical components which were obtained by LIBS. As it can be noticed, the results are consistent. However, the LIBS 
profiling is encumbered with errors with identification of the correct chemical contents on the layers interfaces. Comparing the figures, it can be easily deduced that the ablation rate is slightly lower than $1 \mu \mathrm{m} /$ laser pulse (since carbon disappears after the 2nd pulse and titanium shows up around the 12 th). On the other hand, molybdenum is uncovered earlier than expected - after the 4th laser pulse. It can be attributed to strong beam focusing which results in a Gaussian beam profile and thus not uniform ablation due to which at the center of the crater tungsten is removed faster. Then molybdenum appears to be a material which is even harder to be removed which explains why then 8 consecutive pulses are needed to observe the titanium line. Another problem was with the persistent tungsten presence in spectra even after 16 pulses which was inadequate to its presence or rather its lack at this depth. Again, it seemed to be a problem with non-uniform ablation which led to slow erosion of the tungsten from the crater boundaries.

However, these results might have been considered as partially successful and the problem with the non-uniform layer ablation and, consequently, the chemical profiling distortions needed some consideration. The conclusion was that the problem may be attributed to very strong focusing which was, on the other hand, required for obtaining a power density level necessary for a satisfactory signal intensity in terms of the SNR. It led to the assumption that testing the LIBS in dual pulse (DP) configuration may be worth considering.

The advantage of the DP approach bases on the beneficial influence of a number of phenomena occurring during the interaction of the second laser pulse with the specimen and its vicinity modified by the first pulse. Not only does the second pulse interact with a heated sample but also the interaction occurs in a "bubble" of rarefied gas which stabilizes the plasma and significantly elongates its optical emission. It can be depicted as if the particles ejected from the target in the second pulse collide with those rejected by the first one and thus stay in the observation area for longer (such an explanation is still valid in low-pressure conditions). On the other hand, there are many other mechanisms of the DP enhancement and the majority of them are thoroughly discussed in [24].

\subsection{Dual-pulse LIBS experiments}

Since DP LIBS had been considered to be a method offering a better SNR and dynamics, dual pulse laser LOTIS 3D had been purchased at the IPPLM and the experiments were performed. Indeed, after some optimization works, the results of the measurements performed in atmospheric conditions were spectacular both for tungsten and for stainless steel (which was used in this test in the perspective of its application as the construction material for Wendelstein 7-X stellarator). These results are shown in Figs. 4 and 5.

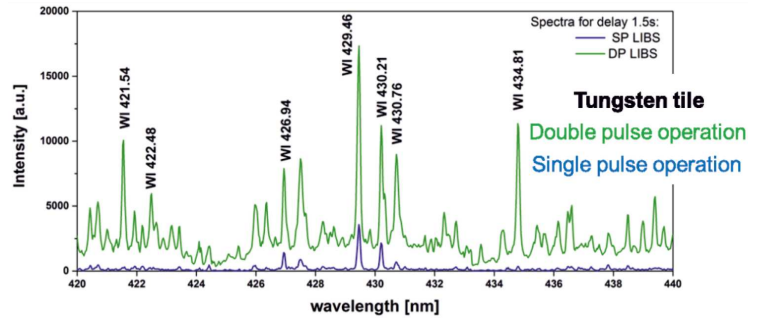

Fig. 4. Comparison of SP and DP LIBS spectra obtained for a tungsten sample.

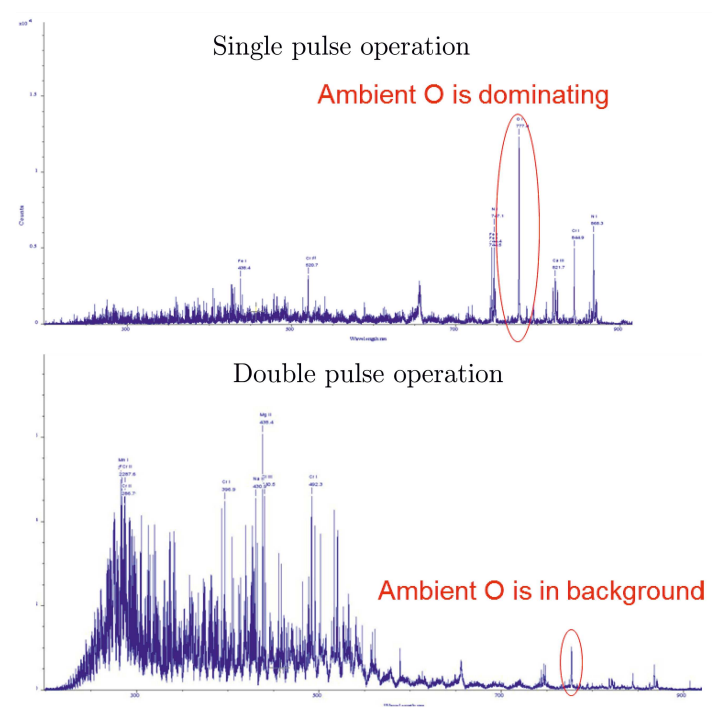

Fig. 5. Comparison of SP and DP LIBS spectra obtained for a stainless steel sample.

Figure 4 shows the SP and DP spectra obtained with $1.5 \mu$ s delay (after the single pulse in SP and after the second one for DP). In the DP spectra, the intensity of the lines is not only significantly higher but the lines are also more numerous. It may be expected that the signal acquired in this way manifests significantly higher metrological value.

A similar phenomenon may be observed in Fig. 5 for stainless steel. What is conspicuous here is that the lines which may be attributed to the atmospheric gases dominating for the single pulse are considerably smaller when compared with the steel components' lines in the DP spectrum.

After this preliminary research, there were many successful attempts in the domain of the thermonuclear fusion research, especially for characterisation of the deuterium/hydrogen release due to subsequent laser pulses. Exemplary results are presented in Fig. 6 which show the deuterium-containing tungsten layer removal from a molybdenum sample. The experiments were performed both in a vacuum $\left(\approx 5 \times 10^{-5}\right.$ mbar $)$ and atmospheric conditions and as an indication of the deuterium contents there was the initial ratio of the deuterium line to the background. The experiments showed that in the atmospheric conditions, the removal process is slightly 


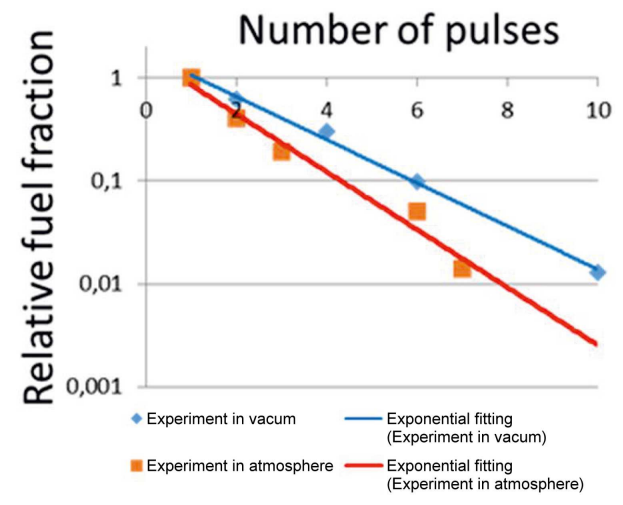

Fig. 6. Comparison of the deuterium contents chemical profiling in a vacuum and in atmosphere.

faster; however, also in a vacuum, the fuel inventory reaches the level $1 \%$ of its original value after 10 laser pulses in comparison with 7 pulses needed for obtaining this level in atmosphere.

In the DP experiments the basic issue was the optimisation of the inter-pulse separation which mostly depends on the pressure and needs to be short in a vacuum, i.e., 50-300 ns, and significantly longer, i.e., $1-10 \mu \mathrm{s}$, in the atmospheric conditions. On the other hand, the plasma evolution in the dual-pulse regime is intricate and for more advanced specimens, as substrates with mixed layers, the optimisation of the inter-pulse separation required empirical study mainly due to different behavior of the layers (heat propagation barriers, delamination) and different chemical compositions (the optimal values depend on the element which is crucial for the analysis).

\subsection{Application of the DP in a tokamak}

The IPPLM activity in the field of LIBS [25, 26] and DP LIBS resulted in close collaboration with ENEA Frascati which facilitated common experiments, first on the calibrated samples, which were described in [27-29], and further on the mockup of the FTU tokamak. The experiment performed on the mock-up of the FTU in autumn 2018 demonstrated a successful operation of the combined broad-band and narrow-band spectrometers for full characterisation of the laser plasma induced from an arbitrary point of a poloidal cross-section of the model. The reason for employing both types of spectrometers was to obtain all the spectral features essential for characterisation of the chemical composition of the sample with the use of the calibrationfree (CF) LIBS. The continuation of these experiments is foreseen for September 2019 and it will include repeating the investigation on the FTU.

\section{Summary}

During the research at the IPPLM, the LIBS method evolved from a simple diagnostics of fuel removal to an independent method for a thorough investigation of the phenomena occurring on the interface of plasma and the wall of the divertor area of the next step fusion reactors. Recent application at the mock-up of FTU and tests of the real machine foreseen for September 2019 prove that the method is ready to be applied in operating tokamaks.

The future of the method should also include the implementation of modern methods of data science such as artificial neural networks (ANN). The first attempts in this area have already been carried out at the IPPLM [30].

\section{Acknowledgments}

This scientific work was supported by Polish Ministry of Science and Higher Education within the framework of the scientific financial resources in the years 2010-2020 allocated for the realization of the international co-financed project. This work has been carried out within the framework of the EUROfusion Consortium and has received funding from the Euratom research and training programme 2014-2018 and 2019-2020 under grant agreement No. 633053. The views and opinions expressed herein do not necessarily reflect those of the European Commission.

\section{References}

[1] I.E. Tamm, Theory of the Magnetic Thermonuclear Reactor, Part I, Plasma Physics and the Problem of Controlled Thermonuclear Reactions, Pergamon, New York 1959, p. 3 (English transl.).

[2] A.D. Sakharov, Theory of the Magnetic Thermonuclear Reactor, Part I, Plasma Physics and the Problem of Controlled Thermonuclear Reactions, Pergamon, New York 1959, p. 20 (English transl.).

[3] R. Aymar, P. Barabaschi, Y. Shimomura, Plasma Phys. Control. Fusion 44, 519 (2002).

[4] J. Roth, E. Tsitrone, A. Loarte et al., J. Nucl. Mater. 390-391, 1 (2009).

[5] T. Hirai, F. Escourbiac, S. CarpentierChouchana et al., Fusion Eng. Des. 88, 1798 (2013).

[6] S. Brezinsek, J.W. Coenen, T. SchwarzSelinger et al., Nucl. Fusion 57, 116041 (2017).

[7] L. Spitzer, Jr., Phys. Fluids 1, 253 (1958).

[8] H. Renner, J. Boscary, V. Erckmann, H. Greuner, H. Grote, J. Sapper, E. Speth, F. Wesner, M. Wanner, Nucl. Fusion 40, 1083 (2000).

[9] D.A. Cremers, L.J. Radziemski, T.R. Loree, Appl. Spectrosc. 38, 721 (1984). 
[10] A.K. Nayak, M.M. Rahman, R. Naidu, B. Dhal, C.K. Swain, A.D. Nayak, R. Tripathi, M. Shahid, M.R. Islam, H. Pathak, Sci. Total Environ. 665, 890 (2019).

[11] M.Z. Martin, M.A. Mayes, K.R. Heal, D.J. Brice, S.D. Wullschleger, Spectrochim. Acta Part B At. Spectrosc. 87, 100 (2013).

[12] D.A. Cremers, M.H. Ebinger, D.D. Breshears, P.J. Unkefer, S.A. Kammerdiener, M.J. Ferris, K.M. Catlett, J.R. Brown, J. Environ. Qual. 30, 2202 (2001).

[13] C. Aragón, J. Campos, J.A. Aguilera, Appl. Spectrosc. 47, 606 (1993).

[14] C. Pan, N. An, Q. Zeng, Q. Wang, S. Wang, X. Du, Appl. Spectrosc. 70, 702 (2016).

[15] J. Liu, W. Ni, X. Li, Y. Fu, Z. Wang, Z. Li, Appl. Spectrosc. 68, 955 (2014).

[16] M. Kurihara, K. Ikeda, Y. Izawa, Y. Deguchi, H. Tarui, Appl. Opt. 42, 6159 (2003).

[17] S.J. Rehse, H. Salimnia, A.W. Miziolek, J. Med. Eng. Technol. 36, 77 (2012).

[18] D. Anglos, V. Detalle, in: Laser-Induced Breakdown Spectroscopy, Eds. S. Musazzi, U. Perini, Springer Series in Optical Sciences, Vol. 182, Springer, Berlin 2014.

[19] S. Maurice, R.C. Wiens, M. Saccoccio et al., Space Sci. Rev. 170, 95 (2012).

[20] P. Gasior, J. Badziak, A. Czarnecka, P. Parys, J. Wołowski, M. Rosiński, Phys. Scr. T123, 99 (2006).
[21] P. Gasior, A. Czarnecka, P. Parys, M. Rosinski, J. Wolowski, J. Hoffman, Czechoslov. J. Phys. 56, B67 (2006).

[22] M. Kubkowska, P. Gasior, M. Rosinski, J. Wolowski, M.J. Sadowski, Europ. Phys. J. D 54, 463 (2009).

[23] P. Gasior, M. Bieda, M. Kubkowska, R. Neu, J. Wolowski, AU Team, Fusion Eng. Des. 86, 1239 (2011).

[24] V.I. Babushok, F.C. DeLucia, J.L. Gottfried, C.A. Munson, A.W. Miziolek, Spectrochim. Acta Part B At. Spectrosc. 61, 999 (2006).

[25] V. Philipps, A. Malaquias, P. Gasior et al., Nucl. Fusion 53, 093002 (2013).

[26] A. Malaquias, V. Philipps, P. Gasior et al., J. Nucl. Mater. 438, S936 (2013).

[27] S. Almaviva, L. Caneve, F. Colao, P. Gasior, M. Kubkowska, M. Łepek, G. Maddaluno, Fusion Eng. Des. 96, 848 (2015).

[28] S. Almaviva, L. Caneve, F. Colao, G. Maddaluno, N. Krawczyk, A. Czarnecka, P. Gasior, M. Kubkowska, M. Lepek, Phys. Scr. T167, 014043 (2016).

[29] R. Fantoni, S. Almaviva, L. Caneve, F. Colao, G. Maddaluno, P. Gasior, M. Kubkowska, Spectrochim. Acta Part B At. Spectrosc. 129, 8 (2017).

[30] M. Łepek, P. Gasior, Proc. SPIE 9290, 929027 (2014). 